\title{
Amplificación, una estrategia de transformación social a partir de la ocupación del espacio acústico.
}

\author{
Abramovici, Gabriel
}

\author{
Gabriel Abramovici \\ gabramovici@psi.uba.ar \\ Hospital Piñero, Argentina \\ Hospital Piñero, Argentina \\ Colectivo85, Argentina \\ Universidad de Buenos Aires, Argentina \\ Universidad Nacional de Lanús, Argentina
}

ECOS - Revista Científica de Musicoterapia y

Disciplinas Afines

Universidad Nacional de La Plata, Argentina

ISSN-e: 2718-6199

Periodicidad: Semestral

vol. 6, núm. Esp.3, e011, 2021

revista.ecos@presi.unlp.edu.ar

Recepción: 27 Julio 2021

Aprobación: 11 Septiembre 2021

URL: http://portal.amelica.org/ameli/

jatsRepo/459/4592620006/index.html

DOI: https://doi.org/10.24215/27186199e011

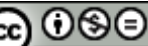

Esta obra está bajo una Licencia Creative Commons AtribuciónNoComercial-SinDerivar 4.0 Internacional.
Resumen: La perspectiva de transformación social implica incertidumbre en cuanto a los cambios que se producirán. Las intervenciones de musicoterapia en el área ecológica pueden tener el foco en los usuarios o en el ambiente. La amplificación como herramienta metodológica permite poner el foco en el ambiente. Se define amplificación como el proceso de presentación a la comunidad de las producciones realizadas en un dispositivo de taller. Sus antecedentes son las performances feministas de la década de 1970, las performances en musicoterapia comunitaria y el campo artístico-cultural de la reforma psiquiátrica brasilera. Su dimensión estética está relacionada con la valoración comunitaria y el protagonismo de la población participante. Su dimensión ética se sustenta en el consentimiento informado y en el cuidado ante las experiencias de presentación. La amplificación también puede ser utilizada como herramienta de investigación, para contrastar de forma empírica la potencia transformadora de una intervención comunitaria.

Palabras clave: amplificación, transformación social, salud comunitaria, radio, salud mental.

Abstract: The social transformation's prospect implies uncertainty about the changes that will take place. Music therapy interventions in ecological area can focus on the users or the environment. Amplification is a methodological tool that allows us to focus on the environment. Amplification is defined as the process of presenting to the community the productions made in a workshop device. Its antecedents are feminist performances of the 1970s, community music therapy performances, and the artistic-cultural field of Brazilian psychiatric reform. Its aesthetic dimension is related to the community evaluation and the prominence of the participating population. Its ethical dimension is based on informed consent and the care with the presentation experiences. Amplification can also be used as a research tool, to empirically contrast of the transformative power in a community intervention.

Keywords: amplification, social transformation, community health, radio, mental health.

Resumo: A perspectiva de transformação social implica incertezas quanto às mudanças que ocorrerão. As intervenções de musicoterapia na área ecológica podem ter como foco os usuários ou o meio ambiente. A amplificação como ferramenta metodológica nos permite focar no meio ambiente. Amplificação é definida como o processo de apresentação à comunidade 
das produções realizadas em um dispositivo de oficina. Seus antecedentes são as performances feministas dos anos 1970, as performances de musicoterapia comunitária e o campo artísticocultural da reforma psiquiátrica brasileira. Sua dimensão estética está relacionada à avaliação comunitaria e ao destaque da população participante. Sua dimensão ética é baseada no consentimento informado e no cuidado nas experiências de apresentação. A amplificação também pode ser usada como uma ferramenta de pesquisa, para contrastar empiricamente o poder transformador de uma intervenção comunitária.

Palavras-chave: amplificação, transformação social, saúde comunitária, rádio, saúde mental.

\section{INTRODUCCIÓN}

“(...) os musicoterapeutas tenemos más que nunca la responsabilidad de elevar y ampliar nuestros horizontes, para efectuar un impacto de ámbito global que llegue más allá de la sala de tratamiento tradicional”'(Boxill, 1997, p.179).

La intención de este trabajo es dar a conocer el recorrido y los avances en la investigación sobre amplificación, una herramienta técnico-metodológica que permite intervenir en el ámbito comunitario desde la perspectiva de la transformación social. Los contextos histórico-geográficos determinan el surgimiento de prácticas y saberes determinados. Por eso se articulará con intervenciones diversas en salud comunitaria desde el Norte y desde el Sur, buscando una metodología para investigar de forma empírica la potencia transformadora de Radio Espacio Abierto.

Radio Espacio Abierto lleva nueve años de funcionamiento ininterrumpido y 339 programas emitidos. Surgió en 2012 por iniciativa de un grupo de personas usuarias de Salud Mental del Hospital Piñero. Lxs musicoterapeutas estamos incluidxs en el equipo interdisciplinario que acompaña la recuperación de pacientes internadxs y realiza el seguimiento ambulatorio post alta. En este encuadre institucional funcionan los talleres del Club Picaso (Dobón et al., 2019). El taller de radio se puede pensar como parte de las incumbencias de lx musicoterapeuta porque se utilizan “...técnicas o procedimientos en los que las experiencias con el sonido y la música operan como mediadores, facilitadores y organizadores de procesos saludables para las personas y su comunidad" (Ley Nacional de Ejercicio Profesional de la Musicoterapia, 2015, Art. 2).

\section{MUSICOTERAPIA Y TRANSFORMACIÓN SOCIAL}

Bruscia $(2000 ; 2016)$ realiza una sistematización a partir de prácticas de diferentes musicoterapeutas de Estados Unidos y Europa, agrupando las prácticas orientadas en salud comunitaria en el área ecológica “... cuyo objetivo principal es la promoción de la salud en el ambiente sociocultural y/o físico” (2016, p.237). Le adjudica gran importancia a esta área debido a su extensión y el grado de cambio resultante. Se la reconoce comoárea relativamente nueva, con prácticas que no se han desarrollado del todo ni han sido apropiadamente investigadas.

En la segunda edición de Definiendo la Musicoterapia, Bruscia (2000, p. 170) se pregunta dónde está puesto el foco del área ecológica: en el cliente (usuarix) o en el ambiente. ¿Está puesto en mejorar la salud del ambiente, modificando aquellos factores que generan problemas en la salud de lxs usuarixs? ¿O en ayudar a lxs usuarixs a lidiar con esos problemas?

En la tercera edición del libro Bruscia (2016) reformuló esta dialéctica, refiriéndose a los modos directos o indirectos en los que se contribuye al cambio social: "En ocasiones se dan cambios terapéuticos en la relación 
del paciente con los contextos sociales y culturales, en otras la terapia contribuye al cambio social o cultural en estos mismos contextos, de maneras directas e indirectas" (Stige, 2002, p. 220, citado por Bruscia, 2016).

En Argentina, las intervenciones de musicoterapia en el ámbito comunitario surgieron en un momento de crisis socioeconómica extrema y estuvieron marcados por una perspectiva de transformación social. Durante el Segundo Encuentro Argentino de Musicoterapia en la Cámara de Diputados, expresamos con el Colectivo85 (2007):

Nuestra sociedad actual se caracteriza por la exclusio\#n de grandes sectores de la poblacio\#n. Este escenario complejo desafi\#a los marcos tradicionales con los cuales los profesionales de la salud interpretamos e intervenimos sobre la realidad. Nos sen\#ala la necesidad de nuevas pra\#cticas profesionales que investiguen e intervengan con el objetivo de producir una transformacio\#n, no solo de las personas y sus recursos internos, sino tambie\#n de las condiciones materiales de vida. En estas pra\#cticas, la transformacio\#n tanto espiritual como material de las personas se produce a partir de las interacciones interpersonales.

Olaechea y Engeli (2007) plantean que el punto de partida para la transformación es el sentimiento de que algo no está bien y debe ser revertido: la exclusión de millones de personas que viven en pésimas condiciones. A partir de esto, proponen una línea de “(...) transformación social hacia la equidad, democratizacio\#n en el acceso y satisfaccio\#n de los derechos de la ciudadanía (...)” (p.18).

¿Cuál es la diferencia entre cambio y transformación? Olaechea y Engeli (2007) consideran que no es la magnitud del impacto lo que determina la diferencia entre cambio y transformación. La distinción tiene que ver con la idea de incertidumbre: “(...) cualquier transformación real es por principio imposible de definir anticipadamente (...) ya que definirla a priori seri\#a limitarla a la capacidad actual de entender el problema, condenándola asi\# a no ser ma\#s que un cambio puntual." (p. 63). El concepto de incertidumbre está relacionado con la participación y la autogestión, pues es la comunidad la que va a orientar el destino incierto de las transformaciones. Incertidumbre también se relaciona con la noción de acontecimiento de Badiou (2015).

La perspectiva de transformación social marca una posición teórica, ética y política en las intervenciones comunitarias, pues no vamos a procurar sólo la adaptación de las personas y los grupos a sus contextos hostiles, ni su inclusión en una sociedad injusta. Vamos a buscar la transformación de ese contexto adverso o al menos del ambiente, la parte del contexto que según Lapalma (2001) se define por la posibilidad de ser modificado durante la intervención.

Sánchez-Vidal (2020) advierte que las intervenciones comunitarias del Norte (Estados Unidos y Europa) tienden a pautar objetivos ma\#s delimitados como la calidad de vida, el empoderamiento de personas y comunidades o la humanizacio\#n de los servicios psicosociales. En cambio, en algunas intervenciones comunitarias latinoamericanas “...aun pervive la búsqueda de una sociedad ma\#s justa, capaz de satisfacer las necesidades ba\#sicas de todos y de superar las situaciones de explotacio\#n, dependencia y colonialismo..." (Sánchez-Vidal, 2020, p.3). El interrogante que postula este autor es acerca de la viabilidad o los medios con los que cuentan estas prácticas para llevar adelante un objetivo tan grande y no quedarse en la mera utopía.

¿Con qué saberes y tecnologías contamos en musicoterapia para la transformación social? En las primeras intervenciones del Colectivo85 $(2007 ; 2008$; 2016; 2018) desarrollamos la idea del hacer musical reflexivo, como herramienta que permite potenciar procesos de participación y organización. A través de las experiencias con la música y el sonido, las comunidades pueden aumentar el nivel de conciencia sobre su historia, sus geografías, sus necesidades y recursos. El hacer musical reflexivo permite construir una percepción global de la situación de la comunidad: colectiva, empática, que integra el pensamiento con las emociones y permite tomar decisiones. Las experiencias musicales y artísticas son herramientas epistémicas (Isla, 2021) que permiten el incremento del conocimiento y comprensión de las necesidades de la comunidad (Moix Martínez, 2006). 
Pero si además de impactar en la conciencia de los grupos involucrados, se busca poner el foco de la intervención en el ambiente ¿con qué saberes y tecnologías contamos? ¿Qué herramientas metodológicas nos permiten aumentar la equidad, la democratización de los recursos y el ejercicio de derechos? Estas preguntas determinaron la necesidad teórica de desarrollar el concepto de amplificación.

\section{TraYectoria DE LA IDEA DE AMPLIFICACión}

Roth (1983) clasifica las performances feministas de la década de 1970 en Estados Unidos en tres tendencias: autobiográfica/narrativa, mística/ritualista y política. Al respecto de esta última, menciona como ejemplo un hecho sucedido en 1971, cuando Women Artistin Revolution (WAR) envió cartas de protesta y realizó tácticas de guerilla contra el Whitney Museum, protestando por la falta de representación femenina en la exhibición anual.

Ansdell, Pavlicevic y Ruud (2004) describen y analizan abordajes en musicoterapia comunitaria por fuera del modelo consensuado. Varios de estos abordajes incluyen perfomances, es decir, presentaciones de las producciones sonoro-musicales por fuera del espacio tradicional de consultorio. Reconocen que estas intervenciones no-convencionales generan preguntas y cuestionamientos sobre el lugar de lxs musicoterapeutas y la metodología utilizada.

Villegas y Mene\#ndez (2011) describen la realización de performances itinerantes en el marco de una intervención comunitaria llevada adelante por el equipo ICMUS, coordinado por Patricia Pellizzari y Flavia Kinigsberg. Se trata de acciones sonoro-corporales en el espacio público, planificadas o improvisadas por el equipo de musicoterapeutas, con el objetivo de establecer contacto y propiciar el diálogo con la población. Villegas y Menéndez (2011) referencian el texto Hacia una definición de Performance de Taylor (s.f.), en el cual performance implica a una acción teatral con contenido político, con principio y final, sin una continuidad en el tiempo.

Isla y Alfonso (2013) llevaron adelante una intervención de largo plazo con jóvenes en situación de vulneración social (problemas con la ley y pobreza) dentro del programa estatal Envión. Durante el proceso de familiarización, la población manifestó la voluntad de armar una banda o conjunto musical. Se trata de una experiencia única por el carácter participativo en la toma de decisiones y la creatividad de la adecuación técnica. Con apoyo del equipo interdisciplinario, se consiguieron recursos materiales y se instauró un sistema de transferencia de tecnología a través de colores para tocar acordes en el teclado y poder acompañar armónicamente las canciones. El repertorio respondía a la identidad musical de la población, fue elegido por los jóvenes y no por el equipo técnico. De forma autogestiva, el ensamble comenzó a presentarse en vivo en fiestas y eventos barriales.

Isla y Alfonso (2013) caracterizaron a este fenómeno de presentaciones en vivo como experiencias performativas. Hay tres elementos novedosos de esta intervención con respecto a la noción de performance de Taylor (s.f.): 1) no se trató de acciones aisladas, sino de un proceso; 2) el sustrato artístico predominante fue musical y no teatral; 3 ) el protagonismo de las presentaciones lo tuvo la población y no el equipo técnico/ profesional.

Amarante et al. (2013) encuentran en el proceso de des-manicomialización brasilero, la emergencia de un conjunto de experiencias mu\#ltiples y diversas en el campo artístico-cultural:

“(...) los conjuntos de carnaval en los que usuarios y profesionales de la salud se mezclan con la comunidad, los conciertos musicales realizados en los lugares frecuentados por el pu\#blico de la ciudad, las presentaciones en teatros, las manifestaciones culturales en conferencias" (p. 288).

Amarante et al. (2013) sostienen que las presentaciones dejan de ser aquellas exhibiciones ti\#picas ofrecidas por las instituciones totales, en las que sus internos exponi\#an a un pu\#blico conformado por familiares y conocidos. Son manifestaciones de usuarios de los servicios en espacios comunes a aquellos en los que los 
artistas consagrados por la opinio\#n pu\#blica ofrecen sus especta\#culos. Caracterizan a estas prácticas como un campo autónomo, diferente del campo psiquiátrico e incluso del campo de la atención psico-social.

Demkura, Alfonso y Isla (2016) realizaron una intervención con mujeres en situación de vulneración social (violencia y pobreza) dentro del programa estatal Ellas Hacen. Durante un proceso de trabajo expresivo y reflexivo, las mujeres compusieron una canción, la grabaron y realizaron un videoclip para su difusión. Este proceso de presentación de las producciones a la comunidad fue definido como la dimensión performativa del hacer musical reflexivo. También comenzamos a pensar como dimensión performativa a la difusión de programas de Radio Espacio Abierto a través de radios comunitarias (Abramovici, 2016).

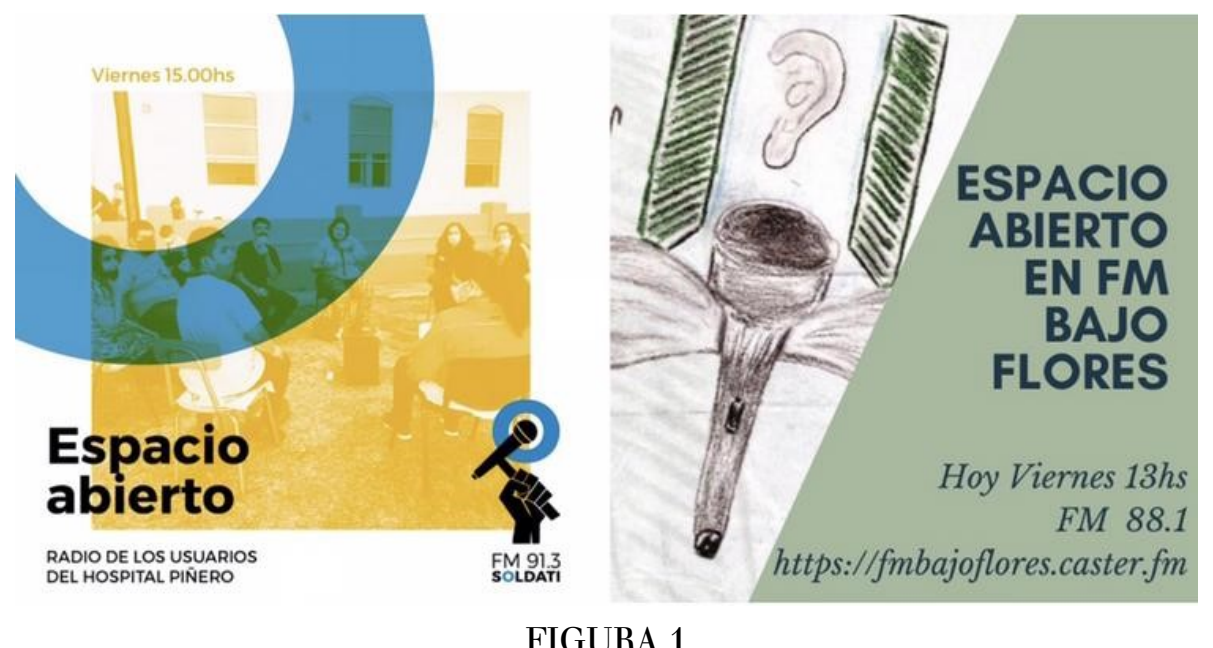

Volantes de difusión de Espacio Abierto Radio por FM Soldati 91.3 y FM Bajo Flores 88.1 (2021)

Taylor (s.f.) advierte que performativo es un falso análogo que se refiere a acciones de la enunciación discursiva. También señala la contradicción de utilizar una palabra de origen anglosajón para referirse a estas prácticas y propone una palabra de origen nahuatl (Ollin). Con el Colectivo85 (2016) consensuamos la utilización de amplificación como equivalente a performance, performático o performativo. Es una palabra en nuestra lengua y remite a un universo que sentimos como propio: el de la música y el sonido

Podemos definir amplificación como el proceso de presentación a la comunidad de las producciones realizadas en un dispositivo de taller. Es diferente de la técnica de empatía descripta por Bruscia (1999) dentro de las 64 técnicas clínicas en musicoterapia de improvisación: "Amplificar: sacar algo que sea distinto o único, sobre la respuesta o comportamiento del paciente y exagerarla” (p.386). Se parece más a la idea de amplificación que plantea Itza (2017) en su trabajo sobre radios de salud mental: "se amplifica, desde la dimensio\#n ma\#s ba\#sica de ampliar, hacer sonar ma\#s fuerte algo que no se escucha" (p.79).

El componente asociado al sonido del concepto amplificación responde a su capacidad para rodear o atravesar obstáculos, ocupar espacios, resonar y recorrer grandes distancias. Especialmente cuando introducimos energía para aumentar la amplitud de las ondas o volumen. Se observa de manera literal cuando organizamos un festival o una radio abierta: sacamos los parlantes al espacio público, intentando que las producciones de un grupo se escuchen fuerte y claro en el ambiente acústico de su comunidad. De la reflexión sobre estas prácticas, surge el concepto de amplificación como proceso participativo de presentación de las producciones. Se nutre de los conceptos de presentación de Badiou (2015) y de reconocimiento (Fraser, 2001; Honeth, 2008; Amarante et al., 2013).

\section{Estrategias de OCUPACIÓN DEL ESPACIO ACÚSTICO}

La amplificación es una herramienta técnico-metodológica que permite poner el foco de las intervenciones en la transformación del ambiente. Las poblaciones silenciadas, excluidas, oprimidas, asumen un rol activo en 
la creación y presentación de producciones a la comunidad, ejercen su derecho a ser escuchadas, reconocidas e incluidas. Esta transformación genera cambios simbólicos y también materiales en las condiciones vida, satisfacción de necesidades y ejercicio de derechos.

Los procesos de amplificación producen cambios en las poblaciones usuarias. Las representaciones cristalizadas se movilizan y se asumen nuevas identidades: de pacientes a comunicadores, de delincuentes a músicos, de jubilados a titiriteros (Los Títeres del Piñero, 2018). El proceso creativo permite desarrollar habilidades intra e interpersonales. Sin embargo, el foco no está puesto en la transformación de los usuarios. La idea de un enunciatario externo, un destinatario de las producciones, es lo que dinamiza estas prácticas.

La amplificación es una estrategia de transformación social a partir de la ocupación del espacio acústico de la comunidad. Es una forma radical de democratizar la expresión buscando el reconocimiento mutuo, forma esencial de subjetivación según Honeth (2008). En las presentaciones en vivo, implica un fuerte compromiso corporal, una militanciaencarnada (Sutton, 2007). En las protestas, movilizaciones, marchas, radios abiertas, el cuerpo funciona como base de las acciones y como texto o argumento político en sí mismo.

Las tecnologías de la información y la comunicación (TIC) forman parte de este espacio acústico. Son una suerte de mediación entre espacio público y los cuerpos. Potencian la amplificación, atravesando barreras de tiempo y de distancia (Cumbre Mundial Sobre la Sociedad de la Informacio\#n, 2004). La Ley argentina de Servicios de Comunicación Audiovisual (No 26.552 / 2009) establece la democratización de los medios de comunicación, pautando espacios destinados a mujeres, pueblos originarios y personas con discapacidad. Al convertir el sonido y la música en ondas radioeléctricas, podemos ocupar el espacio acústico de la comunidad y si nos dejan entrar en los hogares, los autos, auriculares y veredas de la población general.

La idea de amplificación es flexible y permite reflexionar sobre otras intervenciones basadas en la presentación de producciones artísticas, comunicacionales, creativas e incluso comerciales. El desarrollo de la dimensión estética de la amplificación permitió repensar la producción de una empresa social de productos artesanales de lana en Chubut (Carrizo, 2020). La manera particular de escuchar, comprender e intervenir como musicoterapeutas en procesos participativos de producción y presentación, puede ser un aporte de nuestra disciplina a la salud comunitaria.

La amplificación es un proceso muy potente y requiere de una reflexión profunda que garantice prácticas responsables y genuinas. Salir de los dispositivos tradicionales nos deja expuestos a nuevos problemas teóricos. A continuación, intentaremos profundizar en dos de sus dimensiones: estética y ética de la amplificación.

\section{ESTÉTICA DE LA AMPLIFICACIÓN: VALORACIÓN Y PROTAGONISMO}

¿Qué aparece de novedoso a nivel estético? En el modelo clínico, la estética se piensa en términos de verdad dentro del consultorio y no de valor artístico a nivel social. La estética de la clínica es relativa y singular (Rodríguez Espada, 1990; Banfi, 2015). Desde esta perspectiva, cualquier introducción de valores de belleza externos al proceso terapéutico sería un avasallamiento del paciente o grupo. La situación cambia en el ámbito comunitario, donde un grupo puede requerir hacerse escuchar (Kinigsberg, 2011) en su ambiente comunitario. Si intervenimos desde los principios estéticos de la clínica, las producciones organizadas a partir de valores singulares, relativizados, serían difíciles de recibir por la comunidad. El producto puede volverse hermético, bizarro o incluso generar efectos paradojales de re-estigmatización. Se vuelve operativo observar valores estéticos de la comunidad, externos al grupo y a la institución, que funcionen a modo de inclusión. Buscamos que las producciones sean comunicables (Correa-Urquiza, 2009).

El arte y la comunicación no son habilidades exclusivas de un grupo privilegiado. Cualquier persona puede producir hechos artísticos con valor estético (Olaechea y Engeli, 2007). Amarante et al. (2013) afirman que muchas veces se subestima la capacidad de las personas usuarias de salud mental para producir objetos artísticos con valor. La colonización de los saberes de las poblaciones por parte de los saberes profesionales (Correa-Urquiza, 2009) suele impugnar su capacidad para generar producciones valiosas fuera del contexto 
terapéutico. "Las producciones estéticas y culturales de los usuarios de los servicios psiquiátricos demandan ser evaluadas con criterios este\#ticos propiamente dichos...” (Amarante et al., 2013, p. 289).

La amplificación se lleva adelante por decisión de la población participante, no es una técnica que debe ser aplicada. Hay intervenciones muy potentes que no tienen esta dimensión. Balde (2003) realizó un taller de radio coordinado y pensado desde musicoterapia, sin presentar ni publicar las producciones. La exigencia estética debería provenir de la necesidad del grupo participante de ser escuchado y reconocido, no de una presión externa. La búsqueda estética se realiza de forma participativa. Son los participantes quienes escuchan sus producciones de forma crítica y deciden cómo volverlas más comunicables. Boris Groys (2014) afirma que en el arte contemporáneo colapsa la división tradicional del trabajo entre los artistas, que producían las obras, y los curadores, que las seleccionaban y exhibían. La amplificación como proceso participativo, responsabiliza a la población usuaria en la selección de las producciones y en el proceso de exhibición, antes en manos de editores, curadores o bien profesionales y directorxs de instituciones.

Una estrategia bastante utilizada para aumentar el impacto social de la amplificación es la asociación con personas o producciones que ya tengan un reconocimiento social, como artistas o periodistas. Se busca transferir el reconocimiento hacia la población usuaria. Un caso pionero es el trabajo de Stige (1993) basado en la idea de puestos combinados: el musicoterapeuta trabaja en distintos sistemas dentro de la comunidad, por ejemplo un grupo musical y una institución para personas con discapacidad mental y utiliza sus conexiones para establecer redes entre los dos grupos. Algo parecido hacemos en Radio El Encuentro, del Centro de Salud La Otra Base, con el ciclo de entrevistas (Equipo LOBE, 2020).

El problema de este tipo de estrategias es que la población participante puede quedar eclipsada por la producción o el personaje reconocido. Con el fin de tener más audiencia, el grupo de personas que se pretendía visibilizar termina siendo silenciado. Para entender esta paradoja es interesante pensar la oposición de Badiou (2015) entre presentación y re-presentación. Una presentación es una forma singular de mostrarse, otorga efectividad ontológica al ser en su multiplicidad. Se opone a la idea de re-presentación, en la cual esta multiplicidad es anulada por un aparato de poder. En la estética de los procesos de amplificación, la producción de la población usuaria debería ser protagonista. De lo contrario, se perdería el valor singular de la presentación para convertirse en una re-presentación.

\section{ÉTICA DE LA AMPLIFICACIÓN: CONSENTIMIENTO INFORMADO Y CUIDADOS}

¿Cuáles son los problemas éticos que aparecen con la amplificación? La exposición pública es una herramienta poderosa y como tal tiene sus riesgos. Por eso las poblaciones protagonistas deben ser plenamente conscientes de los procesos de amplificación, decidir objetivos y acciones. La ética de la amplificación tiene como principio fundamental el consentimiento informado. Es diferente de la ética de consultorio, centrada en el secreto profesional (nada puede salir de la sala de tratamiento).

La Ley Nacional de Salud Mental (No 26.657 / 2010) fue pionera en materia de derechos y ha influido en el código civil actual (Ley No 26.994 / 2014). A partir de la presunción de capacidad jurídica, todas las personas están capacitadas para tomar decisiones relevantes sobre su vida (Iglesias, 2012). El consentimiento sobre aparición y circulación de producciones en presentaciones públicas o medios de comunicación corresponde a las personas participantes. Sólo un equipo interdisciplinario puede suspender parte de la capacidad jurídica un tiempo limitado, a través de una cautelar como el caso de una internación por salud mental. Los cuestionamientos globales a las prácticas de amplificación provienen del paradigma tutelar en el cual, para salvaguardar la integridad de las personas, se les termina quitando derechos.

Nuestra sociedad cambió mucho en pocos años debido al crecimiento exponencial en el uso de las tecnologías de la información y la comunicación. Surgieron nuevas problemáticas de salud y nuevos riesgos. Con tan solo un celular, cualquier persona puede exponer su intimidad de forma irreversible. A partir de la amplificación, se fortalece a las personas involucradas, explicitando la dialéctica entre lo público y lo privado, 
generando pautas de cuidado de la intimidad y habilidades para manejar las TIC de forma responsable. Por ejemplo, en los talleres de radio, se pueden implementar apoyos y ajustes como la grabación, edición y publicación de programas en dos tiempos, para que las personas que recién se incorporan al proyecto puedan tomar conciencia y decidir qué quieren comunicar. Esto permite que surjan reflexiones críticas de las poblaciones, que pueden empezar a decidir qué mostrar y cómo, qué editar o mejorar para evitar ser vistos o escuchados de una manera indeseable.

Además del consentimiento informado, la ética de la amplificación está relacionada con el acompañamiento y el cuidado de la población usuaria ante las experiencias de presentación pública. Tocar o cantar en un escenario, realizar una performance, hacer títeres en una escuela inicial o hacer radio en vivo, son situaciones muy potentes que deben ser acompañadas con atención por parte del equipo técnico. Para lograr niveles progresivos de confianza y autonomía, hace falta un seguimiento de los efectos que pueden producir las presentaciones en la población usuaria. Está en juego la forma en la que el grupo es percibido por su comunidad. Se pueden reducir estigmas o bien producir efectos paradojales de re-estigmatización y sensación de ridículo. Por eso es importante trabajar participativamente antes, durante y después de la presentación, para consensuar las formas y los contenidos a ser presentados.

\section{LA AMPLIFICACIÓN COMO HERRAMIENTA DE INVESTIGACIÓN}

¿Cómo podemos evaluar o medir la potencia transformadora de los procesos de amplificación? Para Olaechea y Engeli (2007), la transformación social es "una tarea multidimensional, intangible y definitivamente imposible de medir" (p.64). Sin embargo, apostamos a poder dar cuenta del impacto social que tienen la amplificación, con el objetivo de validar y mejorar estas prácticas. La dificultad metodológica reside en dónde ir a buscar los datos empíricos y qué herramientas utilizar. Está en marcha una investigación sobre amplificación de radios de salud mental y reducción del estigma asociado a la locura, dirigida por Martín Correa-Urquiza y Alicia Stolkiner para el Doctorado en Salud Mental de la Universidad de Lanús.

El estigma público que sufren las personas con padecimiento mental produce dificultades en el acceso a la vivienda (Page, 1995; Rodri\#guez, 2014), el empleo (Pickenhagen y Sartorius, 2002; Bordieri y Drehmer, 1986), la participación ciudadana (Ceriani, Obiols y Stolkiner, 2010) y la atención en salud (Vázquez y Stolkiner, 2009). No se trata únicamente de un problema cultural o simbólico, produce estragos a nivel material. Fraser (2007) advierte sobre las relaciones entre luchas culturales por el reconocimiento y luchas por la redistribución económica. Según la autora, el problema es la desconexión entre las dimensiones económica y cultural en las acciones de los movimientos sociales. La transformación social no busca sólo cambios simbólicos o culturales, también pretende la modificación de las condiciones materiales de vida.

Las campañas anti-estigma de origen anglosajón se dividen en educación, contacto y protesta (Corrigan y Penn, 1999). Las radios de usuarios de salud mental surgieron en Argentina con Radio La Colifata, en el año 1991. Son dispositivos anti-estigma que a la vez educan, generan un contacto cotidiano entre usuarios y público, y sirven para llevar adelante acciones de visibilización y protesta en el espacio público. Además, son intervenciones "en primera persona" en las cuales la población habla por sí misma y no mediada por discursos técnicos o profesionales.

Radio Descosidos de Lima (Ponce de León, 2015) y Radio Ábrete Camino de Madrid (Martínez González et al., 2011) se proponen como objetivo luchar contra el estigma asociado a la locura. Radio Nikosia de Barcelona busca de-construir esta imagen negativa o estigma (Correa-Urquiza, 2009). Fortuna y Oliveira (2013) de Radio La Colifata consideran que las radios de salud mental permiten enfrentar a los estigmas y preconceptos sociales y culturales que acompañan a las personas con sufrimiento psíquico. Radio Vilardevoz funciona en Montevideo desde 1997 y tiene como objetivo luchar contra la exclusión y la estigmatización (Baroni, 2019). No se han encontrado investigaciones sobre el impacto de las radios de salud mental en 
relación a la reducción del estigma. Fortuna y Oliveira (2013) se proponen realizar una contrastación empírica, pero aún no lo han logrado.

Bruscia (2016) distingue diversos niveles en las prácticas ecológicas de musicoterapia, de menor a mayor: auxiliares, aumentativas, intensivas y primarias. Sostiene que las prácticas primarias son aquellas que llevan a cambios significativos y duraderos en el individuo o la comunidad y los objetivos y procesos se extienden a través de diversas áreas de prácticas. No refiere ningún antecedente de prácticas primarias en el área ecológica.

Podríamos pensar la Radio Espacio Abierto como una práctica ecológica primaria, porque lleva a cambios significativos y duraderos en las personas (movilización identitaria de pacientes a comunicadores, desarrollo de habilidades intra e interpersonales, inserción educativa y laboral, ejercicio de derechos, autonomía). También lleva a cambios significativos y duraderos en la comunidad (redes con radios comunitarias, organizaciones de usuarios, escuelas de radio, comercios del barrio, actores sociales, instituciones estatales). Y los objetivos y procesos se extienden a través de diversas áreas de prácticas (salud, comunicación, derechos, educación, trabajo, inclusión social y política).

La investigación en marcha tiene como objetivo caracterizar la relación entre la participación en un proceso de amplificación y el estigma público asociado a la locura en los discursos de la población general. Para mantener la coherencia entre teoría y metodología, no se buscarán los datos sobre el impacto de la amplificación en la población usuaria de salud mental, sino en su ambiente. Para ello se utilizará una metodología cuasi-experimental de tipo antes-después. En grupos de discusión con la población general, se realizarán cuestionarios individuales y una discusión colectiva, antes y después de la audición de programas de radio de salud mental especialmente diseñados. Para reducir el efecto Hawtorne, por el cual las personas tienden a responder lo que consideran moralmente correcto y no lo que piensan, se utilizará la técnica de viñetas (Yáñez Gallardo, Ahumada Alvarado y Rivas Aguayo, 2012). Los contenidos a ser escuchados en los grupos de discusión serán producidos y seleccionados por los participantes de Radio Espacio Abierto, quienes están al tanto y participarán como investigadores asociados. La amplificación será utilizada en este caso como herramienta metodológica de investigación. Los resultados podrían aportar a la comprensión de la amplificación como herramienta de transformación social.

\section{Conclusiones}

Amplificación representa a un conjunto de tecnologías y saberes que permiten poner el foco de nuestras intervenciones comunitarias en la transformación social. La estética de la amplificación está relacionada con la valoración de las producciones a partir de lógicas comunitarias, colectivas, en equilibrio con la identidad singular del grupo protagonista. La ética de la amplificación está basada en el consentimiento informado y en el cuidado de la población ante las experiencias de presentación. La amplificación también puede ser utilizada como herramienta de investigación, para realizar una contrastación empírica de la potencia transformadora de una intervención determinada.

\section{Agradecimientos}

Al Colectivo85, por el brillo en los ojos, los debates encendidos y el afecto que nos ha vuelto compadres. Al equipo de la Revista ECOS, por crear, sostener y hacer crecer este espacio de intercambio. A Marcela Bottinelli, por enseñarnos con pasión que se puede investigar lo que creíamos imposible.

\section{ReFERENCiAS}

Abramovici, G. (2016). La radio de usuarios de salud mental como hacer musical reflexivo en musicoterapia, Anais do VI Congreso LatinoAmericano de Musicoterapia, 168-172. CLAM. 
Amarante, P., Freitas, F., Pande, M. R. y Nabuco, E. (2013). El campo artístico-cultural en la reforma psiquiátrica brasileña: el paradigma identitario del reconocimiento. Salud colectiva, 9, 287-299, Universidad de Lanús http: //revistas.unla.edu.ar/saludcolectiva/article/view/194

Ansdell, G.; Pavlicevic, M. y Ruud, E. (2004). Community Music Therapy, Jessica Kingsley Publishers.

Badiou, A. (2015). El ser y el acontecimiento, trad. Cerdeiras, R., Cerletti, A. y Prados, N.. Manantial.

Balde, M. (2003). Voces Emergentes: La práctica musicoterapéutica en el taller de radio de un hospital de emergencias psiquiátricas. Tesina de graduación de la Licenciatura en Musicoterapia de la Universidad Abierta Interamericana (dir. Gustavo Rodríguez Espada).

Banfi, C. (2015). Musicoterapia. Acciones de un pensar estético. Lugar.

Baroni, C. (2019) Una historia de locos: Aportes de Radio Vilardevoz al proceso de desmanicomializacio\#n en Uruguay (1997-2017), Tesis para defender el ti\#tulo de Doctorado opcio\#n Historia, Facultad de Humanidades y Ciencias de la Educación, Universidad de la República, Uruguay.

Bordieri, J. y Drehmer, D. (1986) Hiring decisions for disabled workers: looking at the cause. Journal of Applied Social Psychology, 16, 197-208.

Boxill, E. (1997). The miracle of music therapy. University Park, IL: Barcelona Publishers.

Bruscia, K. (1999). Modelos de Improvisación en Musicoterapia, España: AgrupArte.

Bruscia, K. (2000). Definindo Musicoterapia, Segunda Edicio\#n, Ri\#o de Janeiro, Enelivros.

Bruscia, K. (2016). Definiendo la Musicoterapia, Tercera Edicion. Dallas, TX: Barcelona Publishers.

Carrizo, L. J. (2020). Aportes desde una perspectiva estética de la Musicoterapia a producción artística en la Empresa Social “Quenuir". ECOS - Revista Cientifica De Musicoterapia Y Disciplinas Afines, 5(1), 17-29 https://revista s.unlp.edu.ar/ECOS/article/view/10400

Ceriani, L.; Obiols, J. y Stolkiner A. (2010). Potencialidades y Obstáculos en la Construcción de un Nuevo Actor Social: Las Organizaciones de Usuarios. Jornadas de Investigación Sexto Encuentro de Investigadores en Psicología del MERCOSUR. Facultad de Psicología, Universidad de Buenos Aires.

Colectivo85 (2007). Mu\#sica y comunidad: Accio\#n y reflexio\#n. Demkura, M., Alfonso, S., Isla, C., Abramovici, G. y Morello, R. II Encuentro Argentino de Musicoterapia. Honorable Cámara de Diputados de la Nación.

Colectivo85 (2008). El hacer musical reflexivo: hacia la construcción de una propuesta comunitaria. Abramovici, G., Isla, C., Alfonso, S., Demkura, M., Jiménez, V. y Morello, R. En Abstracts del XII Congreso Mundial de Musicoterapia, p.345. Akadia.

Colectivo85 (2016). El Hacer Musical Reflexivo, una perspectiva en Musicoterapia Comunitaria y Popular. Isla, C.; Demkura, M.; Alfonso, S. y Abramovici, G. En Anais do VI Congreso Latinoamericano de Musicoterapia, 168-172. CLAM.

Colectivo85 (2018). El hacer musical reflexivo. Un aporte desde la musicoterapia orientada en salud comunitaria. Isla, C., Abramovici, G., Demkura, M. y Alfonso, S. En Pellizzari, P. y Rodríguez, R. (dir) Salud, escucha y creatividad: de la musicoterapia preventiva a la musicoterapia social,71-73. EUS.

Correa-Urquiza, M. (2009). Radio Nikosia: La rebelión de los saberes profanos. Otras prácticas, otros territorios para la locura. Tesis Doctoral. Universidad Rovira i Virgili.

Corrigan, P. y Penn, D. (1999) Lessons from social psychology on discrediting psychiatric stigma. The American Psychologist, 54, 765-776.

Cumbre Mundial sobre la Sociedad de la Informacio\#n (2004, 12 de mayo). Declaracio\#n de Principios https://ww w.itu.int/net/wsis/docs/geneva/official/dop-es.html

Demkura, M., Alfonso, S. e Isla, C. (2016). La Dimensio\#n Performativa del Hacer Musical Reflexivo, Relato de una Experiencia con Mujeres. VI Congreso Latino-Americano de Musicoterapia, Brasil.

Dobón, J., Abramovici, G, González, M., Sbriller, L, Schwartz M. (2019). Programa de inclusión a través del Arte, la Socialización y la Ocupación - PICASO II. Premio Mención en las XXXVI Jornadas Multidiciplinarias del Hospital Parmenio T. Piñero, Comité Científico, Gobierno de la Ciudad de Buenos Aires. 
Gabriel Abramovici. Amplificación, una estrategia de transformación social a partir de la ocupació...

Equipo LOBE (2020). Experiencia de Abordaje Territorial Orientada en Reducción de Riesgos y de Daños, Equipo de La Otra Base de Encuentro (LOBE): Abramovici, G.; Barral Santamari\#a, M.; Ciai, S.; Garci\#a Blanco, L.; Gomez Carrillo, P.; Leoz, E.; Olocco, M.; Oviedo, N.; Pelagagge, F.; Remolgado, M.; Solodujin, M.; Venturini, A.; Cuestión Social, Procesos de Organización y Trabajo Social en el Debate Sanitario Contemporáneo, ISSN 2545-8639, 5(1), 180-184, 2020.

Fortuna, D., y Oliveira, V. (2013) Mapeamento das práticas comunicacionais radiofônicas como terapia psicossocial nos serviços de saúde mental no Brasil. Revista Eletrônica de Comunicação, Informação e Inovação em Saúde, 6(4).

Fraser, N. (2001) From redistribution to recognition? Dilemmas of justice in a 'postsocialist' age, en SEIDMAN, S.; ALEXANDER, J. (Orgs.). The new social theory reader, 285-93. Routledge.

Groys, B. (2014). Volverse Público. Las transformaciones del arte en el ágora contemporánea. Caja Negra.

Honneth, A. (2008) Reification, a new look at an old idea. Oxford University Press.

Iglesias, M. (2012). La Reforma al Código Civil Mirando y Mirada por la Convención de los Derechos de las Personas Con Discapacidad. Revista de Salud y Comunidad, 2(2), 67-77, Universidad Nacional de Lanús.

Isla, C. (2021). Arte y Salud Comunitaria. En Isla, C. y Muñoz Rodríguez, M. (comps.) Intervención en salud comunitaria. Relatoria de experiencias [En prensa].

Isla, C. y Alfonso, S. (2013). El ensamble musical como herramienta de intervencio\#n en salud comunitaria, Primer Congreso de Extensio\#n Universitaria, Uruguay.

Itza, B. (2017). Alteraciones y Movimientos. Estrategias de incidencia de Radio Vilardevoz en la construccio\#n de nuevos imaginarios sociales de la locura en Uruguay. Tesis para optar al ti\#tulo de Magister en Psicologi\#a Social. Facultad de Psicología, Universidad de la República.

Kinigsberg, F. (2011). La escucha como produccio\#n de salud, en Pellizzari, P. et al. (2011) Crear Salud, 107-121. Patricia Pellizzari.

Lapalma, A. (2001). El escenario de la intervencio\#n comunitaria. Revista de Psicologi\# a de Universidad de Chile, 10(2). Anuario Comisio\#n de Psicologi\#a Comunitaria. XXVII Congreso Interamericano de Psicologi\#a. SIP.

Ley Nacional de Servicios de Comunicacio\#n Audiovisual nº 26.552, República Argentina (2009) http://servicios.in foleg.gob.ar/infolegInternet/anexos/155000-159999/158649/norma.htm

Ley Nacional de Salud Mental no 26.657, República Argentina (2010) http://servicios.infoleg.gob.ar/infolegInterne t/anexos/175000-179999/175977/norma.htm

Ley Nacional de Ejercicio Profesional de la Musicoterapia no 27.153, República Argentina (2015) http://servicios.in foleg.gob.ar/infolegInternet/anexos/245000-249999/248823/norma.htm

Ley Nacional de Código Civil y Comercial de la Nación no 26.994, República Argentina, (2014) http://servicios.inf oleg.gob.ar/infolegInternet/anexos/235000-239999/235975/norma.htm

Canal Los Títeres del Piñero (2018, 7 de junio) Documental a 10 años de su creación [Archivo de video]. Youtube h ttps://youtu.be/cpM4QNn7Si8

Marti\#nez González, I. et al. (2011) Di\#as de Radio. El esfuerzo hacia la recuperacio\#n: la radio como integracio\#n. Estudos de Psicologia, 16(3), 379-384, Universidade Federal do Rio Grande do Norte.

Moix Martínez, M. (2006). Teoría del trabajo social. Sintesis.

Olaechea, C. y Engeli, G. (2007). Arte y Tranformación Social: Saberes y Prácticas de Crear vale la pena. Buenos Aires: Crear vale la pena. https://www.crearvalelapena.org.ar/img/archivos/5dcead5963e40bfbad7d33e23.pdf

Page, S. (1995) Effects of the mental illness label in 1993: acceptance and rejection in the community. Journal of Health and Social Policy, 7, 61-68.

Pickenhagen, A. y Sartorius, N. (2002) The WP A global programme to reduce the stigma and discrimination because of schizophrenia. World Psychiatric Association.

Ponce de León, J. (2015) Radio descosidos: Una experiencia en salud mental comunitaria con usuarios de un hospital especializado de Lima. Tesis para optar por el título de Magister en Psicología Comunitaria. Pontificia Universidad Católica del Perú. 
Canal Colectivo85 de Musicoterapeutas Comunitarios (2016) Radio Espacio Abierto - Evaluación Participativa [Archivo de video]. Youtube. https://youtu.be/_O1192XJJSo

Rodríguez Espada, G. (1990). Ética y Estética en Musicoterapia. Buenos Aires: Anuario AmuRA.

Rodri\#guez, M. (2014). Universidad, clases y poli\#ticas populares de ha\#bitat en el contexto de la ciudad neoliberal en Habitar en contextos de desigualdad. Proyecto Habitar.

Roth, M. (1983). The Amazing Decade: Women and Performance Art in America: 1970-1980. Astro Artz.

Sánchez-Vidal, A. (2020). Psicología Comunitaria. Definición y Bases Teóricas: Comunidad, Desarrollo Humano y Empoderamiento, Universidad de Barcelona. https://www.researchgate.net/publication/344648969_PSICOL OGIA_COMUNITARIA_DEFINICION_Y_BASES_TEORICAS_COMUNIDAD_DESARROLLO_H UMANO_Y_EMPODERAMIENTO_Alipio_Sanchez_Vidal_Universidad_de_Barcelona

Stige, B. (1993). Music therapy as cultural engagement. Presentado en el Séptico Congreso Mundial de Musicoterapia, España.

Stige, B. (2002). Culture-centered music therapy. Barcelona Publishers. Citado en Bruscia (2016).

Sutton, B. (2007). Poner el Cuerpo: Women's Embodiment and Political Resistance in Argentina. Latin American Politics and Society, 49 (3), $129-162$.

Taylor, D. (s.f.). Hacia una definición de Performance. Fuentes, M. (trad.) Performanceología. http://performancelog ia.blogspot.com/2007/08/hacia-una-definicin-de-performance.html

Vázquez, A. y Stolkiner, A. (2009). Procesos de estigma y exclusión en salud: Articulaciones entre estigmatización, derechos ciudadanos, uso de drogas y drogadependencia. Anuario de investigaciones, 16, 295-303.

Villegas, J. y Mene\#ndez, V. (2011). Grupos focales y performances itinerantes, en Pellizzari, P. et al. (2011) Crear Salud, 154-170. Patricia Pellizzari.

Yáñez Gallardo, R.; Ahumada Alvarado, H. y Rivas Aguayo, E. (2012). La técnica de viñeta y su aplicación en investigaciones en enfermería. Ciencia y enfermería, 18(3), 9-15. 